\title{
BARTER ONLINE NETWORK
}

VAN NGOC TRAN

Barter is the direct exchange of goods or services without using a medium of exchange, such as money. Barter faced a number of limitations, and according to Smith (1776), these limitations led to the emergence of money. However, trading with money also exposes traders to the problems of monetary economy such as inflation, deflation, currency devaluation, and currency exchange fluctuation. According to Statista.com (2015), in 2016, global Business to Customer (B2C) e-commerce sales will reach 1.92 trillion US dollars. On the other hand, online barter solutions are rare on the market. The only attempts to tackle online barter are mobile applications, carried out by small businesses. The market gap is caused by the unsolved inefficiencies of barter. The aim of this thesis is to identify the problems of barter, propose an IT solution for the problems of barter, and finally, produce an artefact, which is the realisation of the proposed IT solution by utilising computer systems and computer algorithms. 


\section{INTRODUCTION}

Before money, there was bartering. According to a journal article by Dalton (1982, p. 181), barter stands for a moneyless market exchange. Barter is defined as a direct exchange of goods and services without using a medium of exchange such as money. Barter inefficiencies are the problems of barter. These inefficiencies will be defined and solved via a proposed model.

\section{PROBLEM DEFINITION: BARTER INEFFICIENCIES}

The first problem of barter is the coincidence of wants. Coincidence of wants in barter is described by Jevons (1875) as "the first difficulty in barter is to find two persons whose disposable possessions mutually suit each other's wants. There may be many people wanting, and many possessing those things wanted; but to allow of an act of barter, there must be a double coincidence, which will rarely happen." The coincidence of wants problem is a transaction cost which imposes limitations on trade under a barter system. To understand how severe this limitation is, let us examine how trade works under a traditional barter scenario (non-electronic barter).

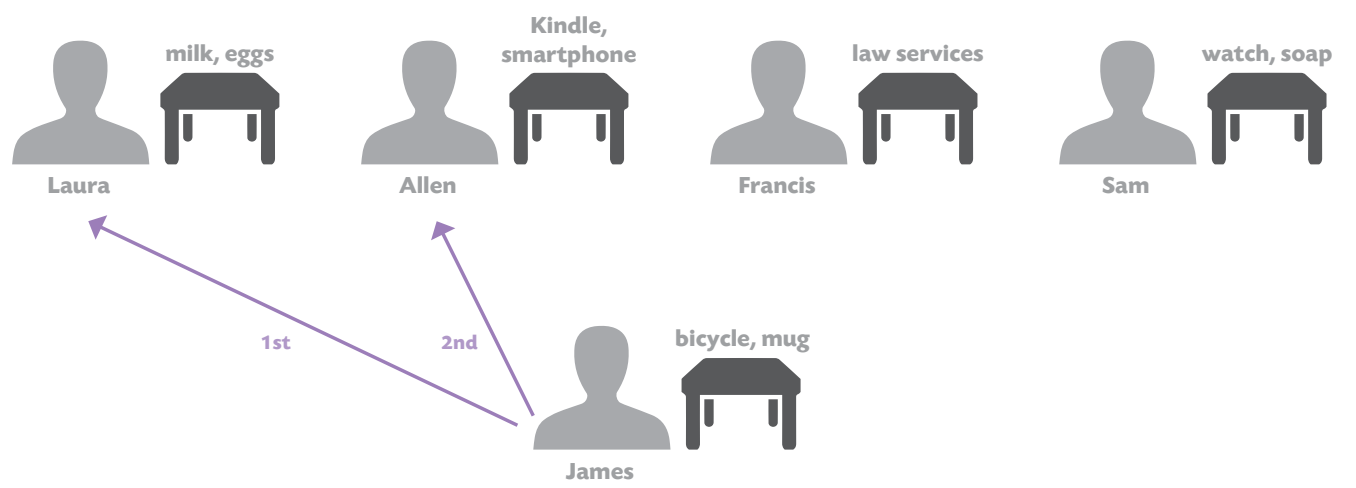

Figure 2.1: Trade under tradition barter scenarios

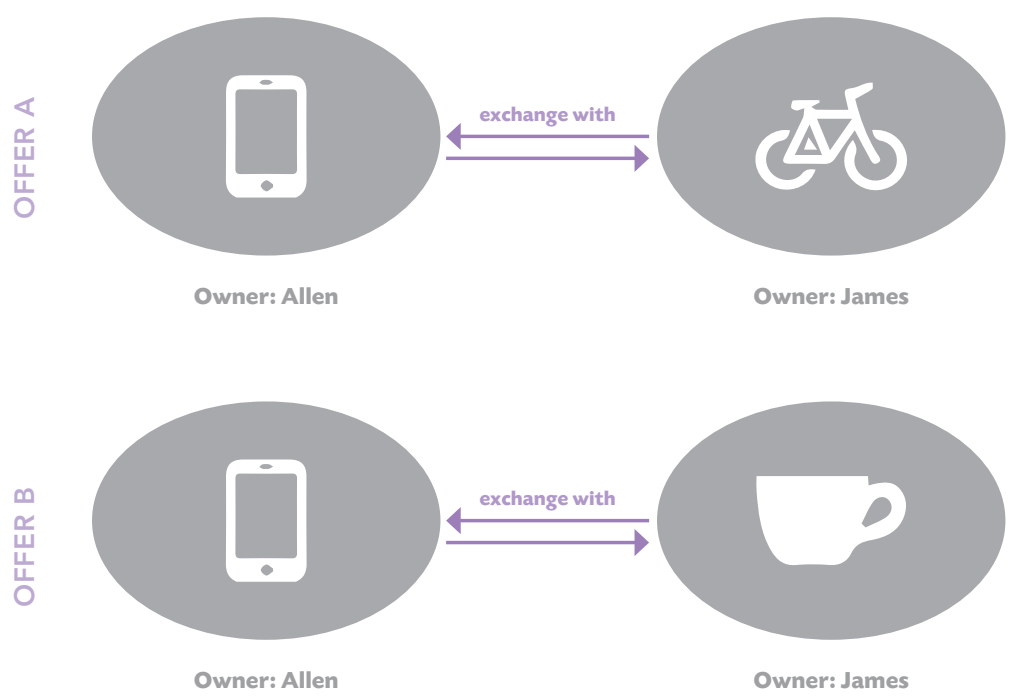

Figure 2.2: Barter offers from James to Allen 
In a traditional barter scenario, trades happen in a physical market, as seen in Figure 2.1. People who wish to trade join the market with items they have. Let us follow the example seen in Figure 2.1. James wishes to trade, hence James joins the market with his items. James must go to one seller at a time to examine what they are selling and only when James identifies an item he wants, James then can offer an exchange with one of his items in return. First, James visits Laura. James is not interested in Laura's items. James then visits Allen. Suppose James finds an item he wants, which is the smartphone Allen is selling. James then offers Allen to exchange the smartphone for one of James' items. The two offers James makes to Allen are visualised in Figure 2.2.

For James, these offers are based on an identified need. James has seen, examined, and decided that he wants one of Allen's items. On the other hand, Allen sees James' items for the first time, thus it is possible that Allen could agree with one of James' offers, but it is also possible that Allen will not agree with any offers made by James. This is the problem of coincidence of wants. Whether Allen agrees to an exchange or not, is a matter or coincidence. As James goes around the market, making offers to other sellers, but there are no guarantees that an exchange will happen.

The second problem of barter is that multilateral trade is less feasible. Barter can be bilateral as well as multilateral. Bilateral barter is an exchange between two parties, visualised in Figure 2.3. User A wants an item of User B and User B wants an item of User A. Multilateral barter is an exchange between multiple parties (more than two), visualised in Figure 2.4. User A wants an item of User B, but User B doesn't want item of User A. However, User B wants an item of User C, and User C happens to want an item of User A, making it a full circle of wants.

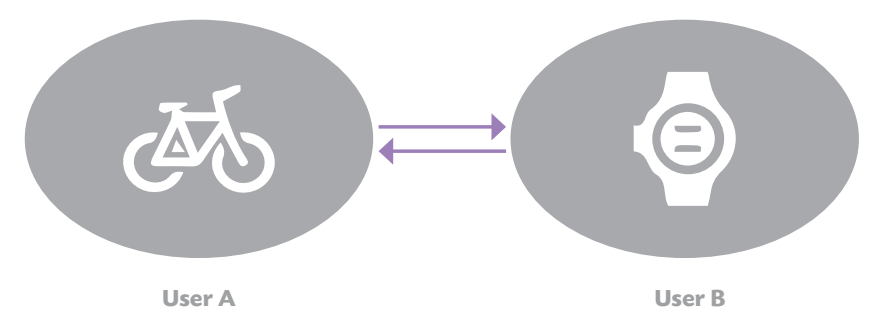

Figure 2.3: Bilateral exchange

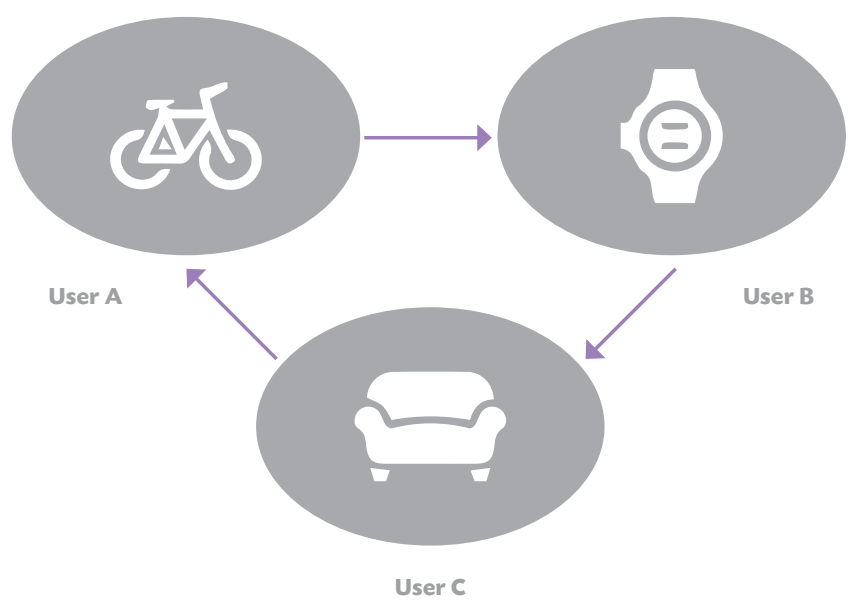

Figure 2.4: Multilateral exchange 
Why is multilateral trade important? When using money to trade, multilateral trade happens regularly. Examine the following example. James is a doctor. James treats a patient and gets paid in return. James then buys bread from a baker and pays with his money. The baker then uses the money to pay for his car wash. The owner of the car wash service then uses the money to pay his rent to the landlord. This is a trade between multiple parties - a patient, a doctor, a baker, a car wash services owner, and a landlord. If trade was limited only to two parties, then right at the beginning, James cannot treat the patient if the patient does not have something James want in return. This would be a major inefficiency.

In barter, as the number of parties involved in an exchange grows, the problem of coincidence of wants aggregates, thus making multilateral trade less feasible than bilateral trade. This is a major inefficiency that needs to be solved.

The third problem of barter is inefficient shopping experience. When shopping using money, the shopping flow is separated into two modules. In the first module, the shopper browses all of the items available in a shop and chooses what he/she wants. In the second module, the shopper makes a transaction by paying with money. When bartering, the shopping experience can be also divided into two modules: browsing/picking items and making a transaction. However, when bartering, these modules are repeated every time the shopper visits a different seller. Another inefficient shopping experience is that in a physical market, sellers can communicate with another seller one at the time. For example, while James communicates with Laura, no one can approach James to check out his items. This means that in a market full of sellers, many people might have to wait in line until a person becomes available. Finally, another inefficient shopping experience is that items are displayed per seller, and not based on its functionality. All of these problems cause barter to be time consuming. It is concluded that the identified problems are: coincidence of wants, less feasible multilateral trade, and inefficient shopping experience.

\section{PROBLEM SOLVING PROCESSES}

The problem solving processes show the construction of how each identified problem was solved. First, problem solving techniques in computer science were chosen to set a general approach to solving problems. The techniques utilised are divide and conquer and abductive reasoning. The divide and conquer strategy decomposes a problem into subtasks. These subtasks are then conquered by abductive reasoning, which uses logic and reasoning to find the simplest and most likely solution to the problem.

The identified problems - coincidence of wants, less feasible multilateral trade, and inefficient shopping experience, were solved using the following processes:

1. Analyse the problem - understand what the problem is about.

2. Decompose the problem - use the divide and conquer technique to decompose the problem into subtasks.

3. Solve each subtask - find the simplest and most likely solution to the problem.

4. Develop an algorithm (optional) - develop an algorithm to solve a problem based on its solutions. The algorithm contains a step-by-step set of operations.

5. Check algorithm efficiency (optional) - calculate algorithm efficiency.

6. Reflect on the whole process - conclude in findings.

Steps 4 and 5 are optional. An algorithm is developed only when the solution demands a computer algorithm to solve a problem. The following sections show how each identified problem was solved. 


\subsection{COINCIDENCE OF WANTS}

The first problem to be solved is coincidence of wants, which is defined as the difficulty in barter to find two persons whose disposable possessions mutually suit each other's wants. The problem can be understood as:

- Scenario: there are two people whose item mutually suits each other's wants.

- Problem: it is difficult to find (match) these people.

Let us decompose the problem into subtasks (see Table 3.1). Let us call the two people whose item mutually suit each other's wants as person A and person B.

\begin{tabular}{|l|l|}
\hline Subtask & Problem \\
\hline \#1 & $\begin{array}{l}\text { How does person A find and show interest in an item of person B? How does person B find and show inter- } \\
\text { est in an item of person A? }\end{array}$ \\
\hline \#2 & $\begin{array}{l}\text { How can person A and person B efficiently match their items that mutually suits each other's wants (if the } \\
\text { market is composed of x number of sellers)? }\end{array}$ \\
\hline
\end{tabular}

Table 3.1: Subtasks to the problem of coincidence of wants

Let us find the simplest solutions to these subtasks.

Subtask \#1 poses the same question, only from the perspective of two different people. The question can be abstracted to "how does any person find an item and show interest in an item of any other person?" The simplest solution to the problem is for the IT solution to collect all of the information of all the people, items, and interests into a database. Any person then can find any item belonging to any user in a table which collects data about items. When a person shows interest in an item, the interest is recorded in the interest table. Any software depends on a database. A database is a collection of data. The solution to build a database, which collects and organise all data necessary for barter, is concluded as the simplest and the most likely solution to the problem.

Subtask \#2 poses the question "how can person A and person B efficiently match their items as mutually suiting each other's wants (if the market is composed of x number of sellers)?"

The key to understanding this question is the keyword ' efficiently', which means that the solution, aside from solving the problem, must also provide efficiency. The simplest solution to this problem is to develop a computer algorithm to match an item of person $A$ and an item of person $B$ that mutually suits each other's wants. As information about people, items, and interests are collected into a database, a computer algorithm can be developed to take the collected data as input and proceed to find matches of mutual wants. The purpose of a computer algorithm is to effectively calculate a task. The solution of using a computer algorithm for a calculation of tasks instead of leaving the calculation to humans is justified as humans are prone to error and the size of a database of a typical software is not feasible for a human to process. It is concluded that the solution is the simplest and the most likely solution to the problem.

\subsection{MULTILATERAL TRADE FEASIBILITY}

The second problem to be solved is that multilateral trade is less feasible than bilateral trade, due to the problem of coincidence of wants aggregating as the number of participants grows within a barter transaction. The root cause of the problem is the problem of coincidence of wants. If this root cause is solved, the problem is solved. The problem of coincidence of wants has been already solved, thus we already have a solution to the problem of multilateral trade feasibility. 


\subsection{INEFFICIENT SHOPPING EXPERIENCE}

The third problem of barter is inefficient shopping experience, which includes: repetition of tasks as a person move of one seller to another seller, one seller can communicate with another seller one at the time, and items are displayed per seller, not based on the functionality of an item. These problems cause barter to be more time consuming than shopping with money. Let us decompose the problems into subtasks (see Table 3.2)

\begin{tabular}{|l|l|}
\hline Subtask & Problem \\
\hline \#1 & How can a person avoid repeating the same barter steps every time the person visits a different seller? \\
\hline \#2 & How can a person avoid being limited to bartering with one person at a time? \\
\hline \#3 & How can a person browse items based on functionality of the item rather than to whom the item belongs to? \\
\hline
\end{tabular}

Table 3.2: Subtasks of the problem of inefficient shopping experience

Subtask \#1 poses the question "how can a person avoid repeating the same barter steps every time the person visits a different seller?"

The simplest solution to this problem is for the IT solution to break the barter process into stages that mimics the shopping experience. The stages are:

1. Person $X$ browses all items.

2. Person $X$ shows interest in items.

3. A computer algorithm finds mutual wants (internal step, performed by the IT solution itself)

4. Person $X$ makes a barter transaction by: browsing mutual wants, choosing what he/she likes, and performing the barter.

Subtask \#2 poses the question "how can a person avoid being limited to bartering with one person at a time?"

The solution to Subtask \#1 already solves the problem of Subtask \#2. By breaking the barter process into the above mentioned stages, a person is not limited to another person one at the time. Instead, any person can browse all items at any time, no matter who is the owner. Any person should be able to show interest in any item as well.

Subtask \#3 poses the question "how can a person browse items based on functionality of the item rather than to whom the item belongs to?"

The simplest solution to the problem is to display items to people based on functionality (just as items in a shop are displayed based on its category) rather than based who owns them. The IT solution will be consisted of a presentation layer, where data is fetched from the database but presented to people based on a set of business logic. Display items to people based on functionality shall be one of the business logic aspects.

It is concluded that mimicking a standard shopping experience is the simplest and most likely solution to the problems. 
The proposed model is an electronic barter (e-barter) system, hereinafter referred to as the system. The system is a barter network of people who wish to trade products or services for other products or services, hereinafter referred to as an item. The system implements the solutions to the problems of barter inefficiency. The system access point shall be a web application, accessible through desktop computers and mobile devices. The back-end is consisted of a database. The objective is to showcase the system as a proof of concept.

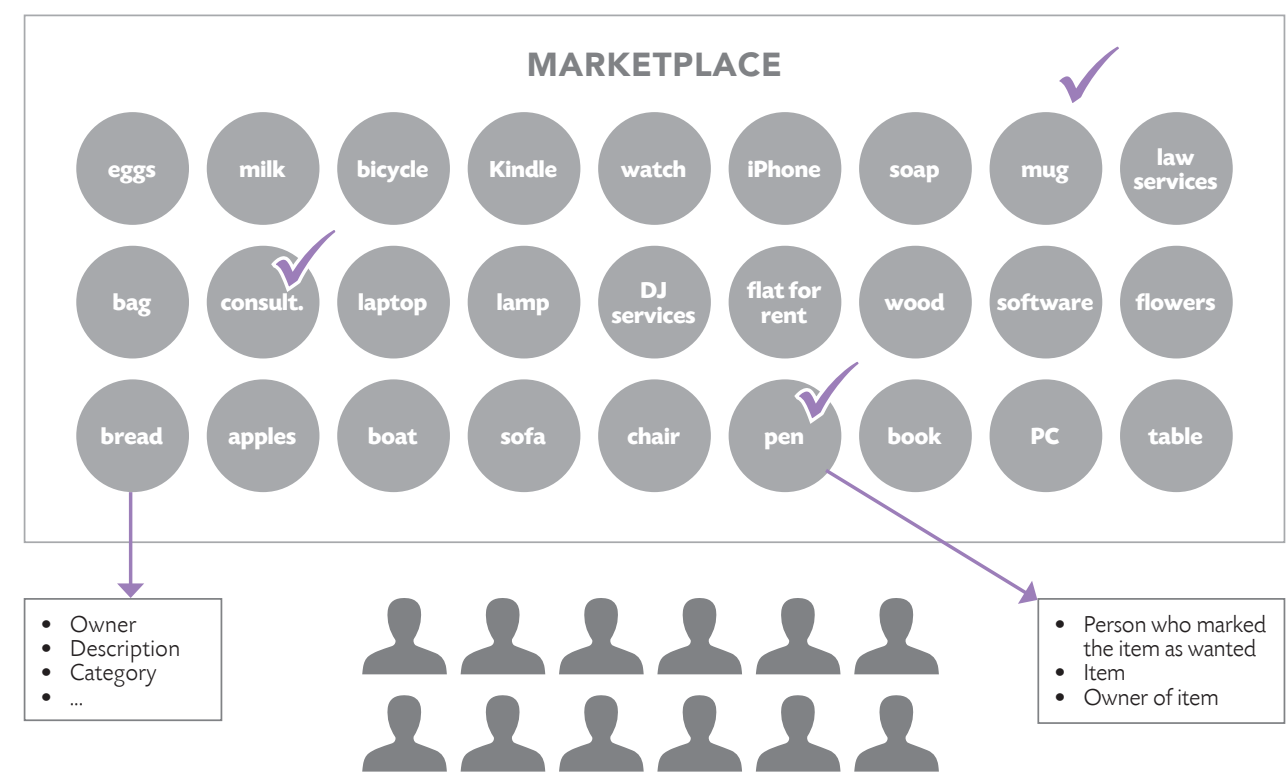

REGISTERED USERS

Figure 4.1: Proposed model - marketplace

Just like a physical barter market, the system is consisted of participants, referred to as users. Anyone can become a user by registering into the system using their email address.

Just like a physical barter market, a user has items. The first shift from the traditional barter scenario is that here, users' items are not presented on separate tables belonging to individual sellers; instead they are presented together in a pool of items. This is called a marketplace, as visualised in Figure 4.1. The marketplace is comprised of all items from all users. Each item contains information such as: owner of the item, title, description, category, subcategory, and images. The marketplace serves one purpose, which is getting users identify their items of interest by letting them find and mark any items they want as wanted. The marketplace shall have search and filtering functionalities, making it more convenient for users to find a specific item under specific criteria. The introduction of the marketplace removes identified problems of barter described in Chapter 2 by:

- Making shopping experience modular. A user does not need to complete all phases of trade before moving to another seller. The marketplace resembles a typical shop, where users pick items they want, regardless of who owns the items and what they have to offer in return.

- Making the shopping experience more efficient due to the search and filter functionalities (items can be displayed based on their functionality).

- The virtual market also lifts the restraint of sellers only communicating with each other one at a time, as seen in a physical market. 
When a user marks an item as wanted (represented as a checkmark in Figure 4.1), the system collects a buy request. A buy request contains information, such as the person who marked the item as wanted, the item itself, and the owner of the item. From this table of information, the system shall run the algorithm to find items of mutual wants. Mutual wants can be in one-to-one, one-to-many or many-to-many relationship, visualised in Figure 4.2.
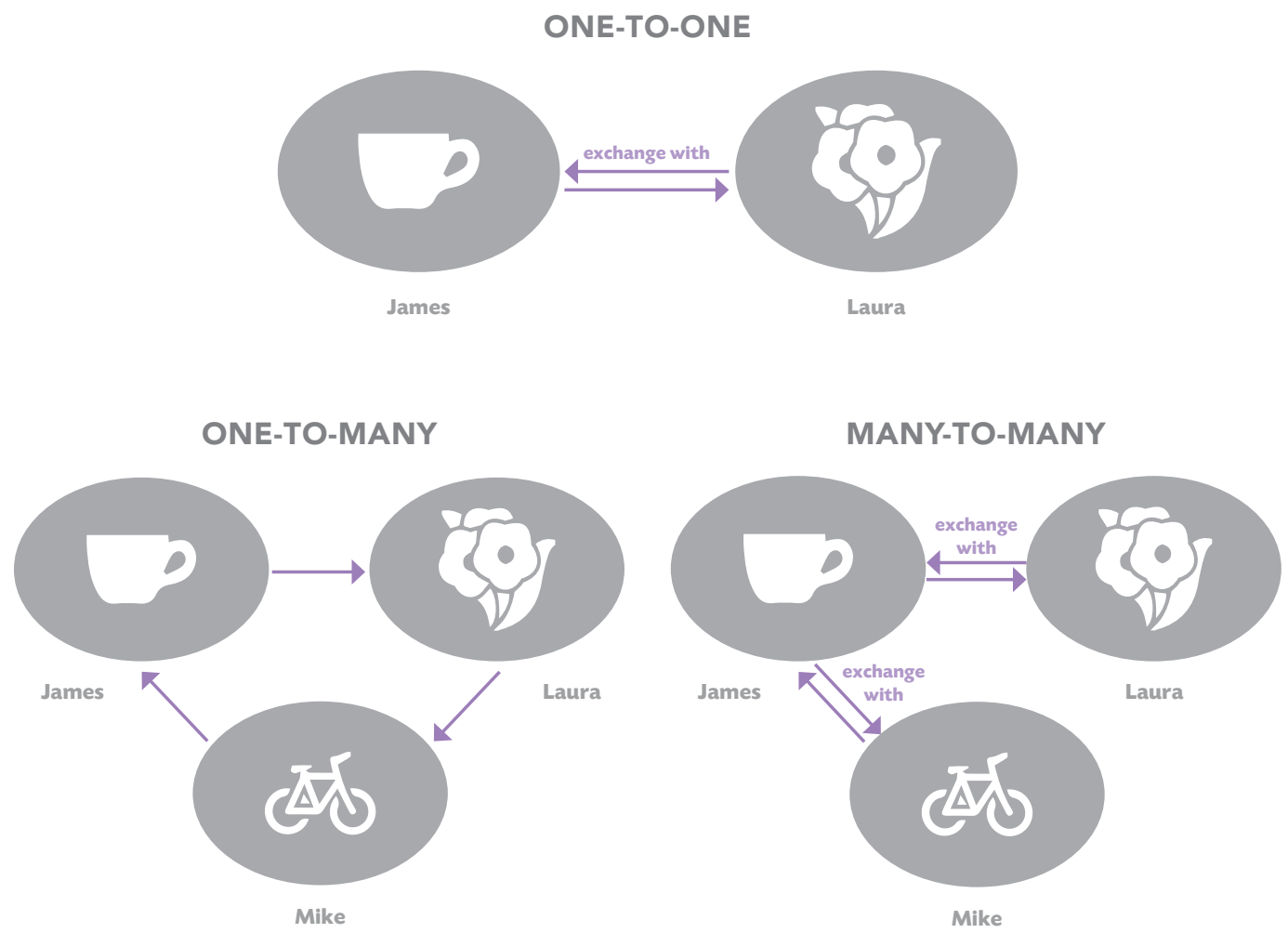

Figure 4.2: Mutual wants

These mutual wants found by the system are barter options, which are presented to a user in his/her shopping cart. For each of his/her item, barter option/s will be shown. These barter options are no longer dependent on coincidence of wants; rather each option consists of identified wants. For example, supposed James and Laura find a barter option seen in Figure 4.2 (one-to-one example) in their shopping cart. This option can tell us that James marked item flowers from Laura as wanted and Laura marked item mug from James as wanted. By letting the system find mutual wants based on users' expressed interest, another problem of barter, coincidence of wants, is removed.

When a user picks an option, a transaction is created. A transaction is considered completed when all parties involved in the exchange picked the same option and all parties involved in the exchange performed handover their items. An optional step in the transaction is rating the item received.

The problems of barter identified in Chapter 2 have been tackled and removed. Barter in the proposed model is based on identified wants. As the problem of coincidence of wants was removed, multilateral exchanges are feasible and increase the probability of exchanges occurring. Lastly, the business logic and proposed features improve the barter shopping experience. 


\subsection{DATA LAYER DESIGN}

The database design is seen in Figure 5.1.

The Proposed Model was reviewed to determine what information is needed to be collected. Data which can form real-world objects were identified and then turned into tables. These tables are seen as full-lined in Figure 5.1. Each row of data in these tables represent an elementary object, meaning not further decomposable. This ensures that when the database is being expanded, it expands outwards, and existing tables will not have to be broken down into more tables. Relationships between these tables were identified and junction tables were created, wherever necessary. Junction tables are seen as dotted-lined in Figure 5.1.

The database is then checked for violations of database normal forms; this process is called database normalisation. Database normalisation is the process of organising data in relational databases, which reduces or eliminates data redundancy and prevent insert, update, and deletion anomalies. The database is in First Normal Form (1NF):

- Each table has a unique identifier in the form of primary key.

- Each field contains one value.

- No repeating groups of columns.

The database is in Second Normal Form (2NF), which means that any non-key fields are dependent on the entire primary key. No tables uses compound keys, thus the $2 \mathrm{NF}$ is satisfied.

The database is in Third Normal Form (3NF), which states that no non-key fields are dependent on another non-key field. No tables violate the 3NF.

The UML has been colour-coded to show the four entities (an entity refers to tables which are in a close relations with each other), which make up the database.

\subsection{BUSINESS LOGIC LAYER DESIGN}

The business logic layer concerns with the retrieval, processing, transformation, and management of application data and the application of business rules. The business logic layer consists of:

- Business entities, which are business objects that represent real world elements, such as login, customers, or orders.

- Business workflows, which are patterns of a user's activity, dictating how a user's tasks are completed to satisfy business process and rules.

The proposed model and software requirement specification were examined and the business entities have been designed to reflect these requirements. The application is consisted of the following entities: Register, Login, My Profile, Profile, My Items, Marketplace, My Cart, and My Transactions.

The Register entity concerns with user registration. An unregistered user must register in order to access the application. A user registers via a form, filling in their name, email address, and password. After registering, the user can immediately log into the system. 


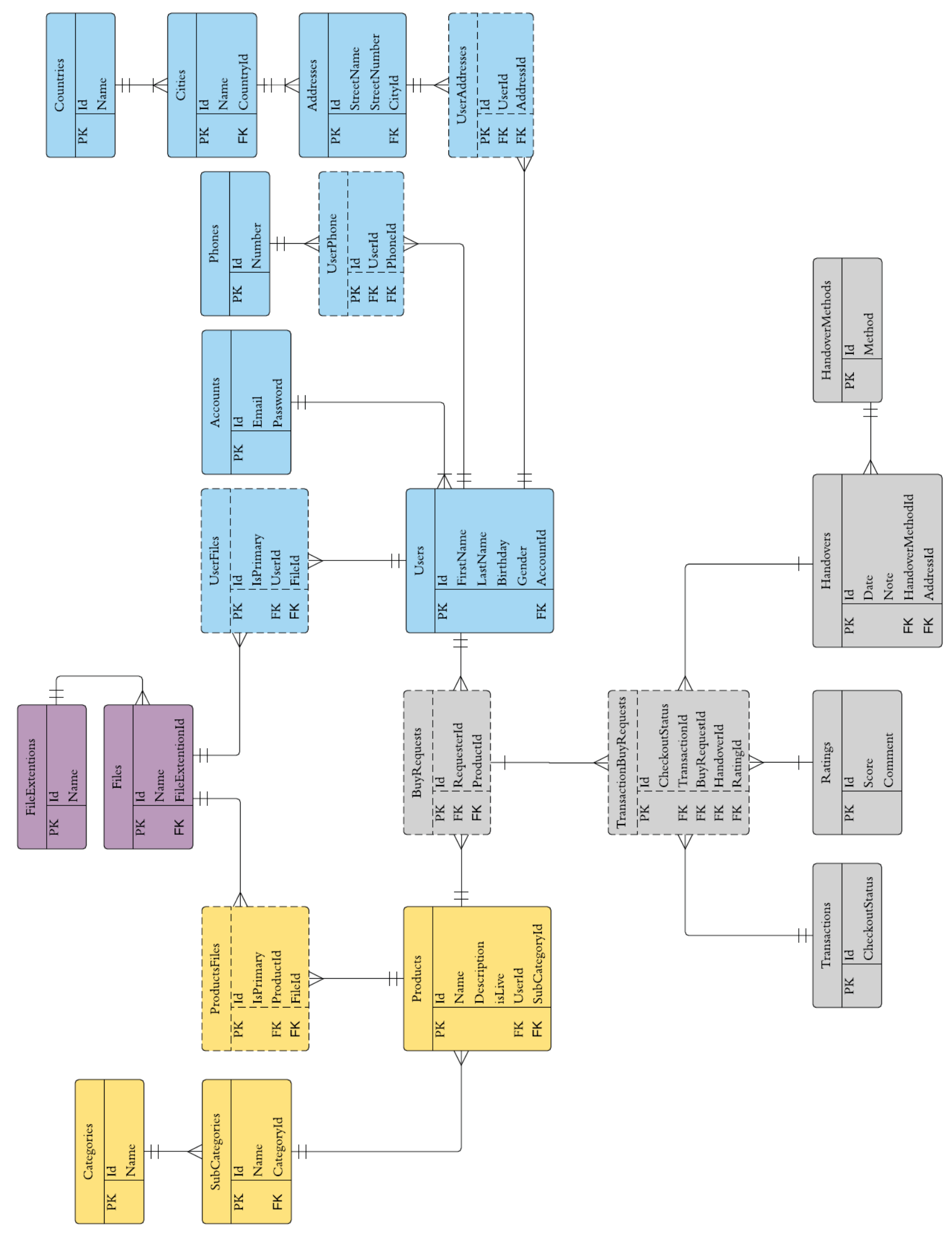

Figure 5.1 Database design, UM 
The Login entity is concerned with user login and logout processes. A user can login to the system, by providing user credentials, as well as log out of the system. While logged into the system, the user is associated with an ID and can perform any of the functionalities offered by the application while being recognised as a particular user.

The My Profile entity deals with the profile of the logged in user. User profile stores personal information and account information. A user profile is public, thus accessible to both the owner and other users. A user should be able to edit their profile.

The Profile entity deals with the profiles of other users. All profiles are public, thus any user can view the profile of other users; however, only with view permission. Profile shows basic information, the items belonging to that particular user and how the user is rated.

The My Items entity is concerned with the management of users' items. The My Item entity shows the logged-in user their items, and the user can perform CRUD actions on these items, such as add, edit, view, and delete items. When individual items are viewed, the user should be able to see a list of people who marked the item as wanted.

The Marketplace entity is concerned with the display of items of other sellers. The one action a user can perform is to mark any of these items as wanted, as well as undo the action.

The My Cart entity is concerned with the display of barter options, found by the system algorithm. My cart will list all the items which are ready to be checked out. There can be one barter option or multiple barter options for each item. The user then must checkout (pick) one option to start the transaction process.

The My Transaction entity concerns with the display of user transactions. When a particular transaction is viewed, we shall see a three step process: accept transaction, handover items, and rate transaction. A transaction is considered finished if all of the participants within a transaction finished the first two steps. The third step - rating - is an option.

By examining the business entities, the business workflow has been worked out as seen in Figure 5.2.

\subsection{PRESENTATION LAYER DESIGN}

In order to design the presentation layer, the application type must be determined. The proposed model established that the application type shall be a web application. To satisfy the requirement of the application being accessible via desktop computers and mobile devices, a responsive web design approach will be utilised. The responsive web design technique adapts application layout to any device's resolution, hence providing multi-device experience across smartphones, tablets, laptops, and desktop computers while running a single web application (as opposed to having a separate web version and a separate mobile version).

The presentation layer is the access point for users. The user interacts with the system via the application $\mathrm{UI}$, consisted of UI components, which accept user input and controls what information is display to the user. The UI has been designed to maximise user experience, as well as to reflect the requirements in Chapter 2. Some of the UI designs are seen in the next pages. 


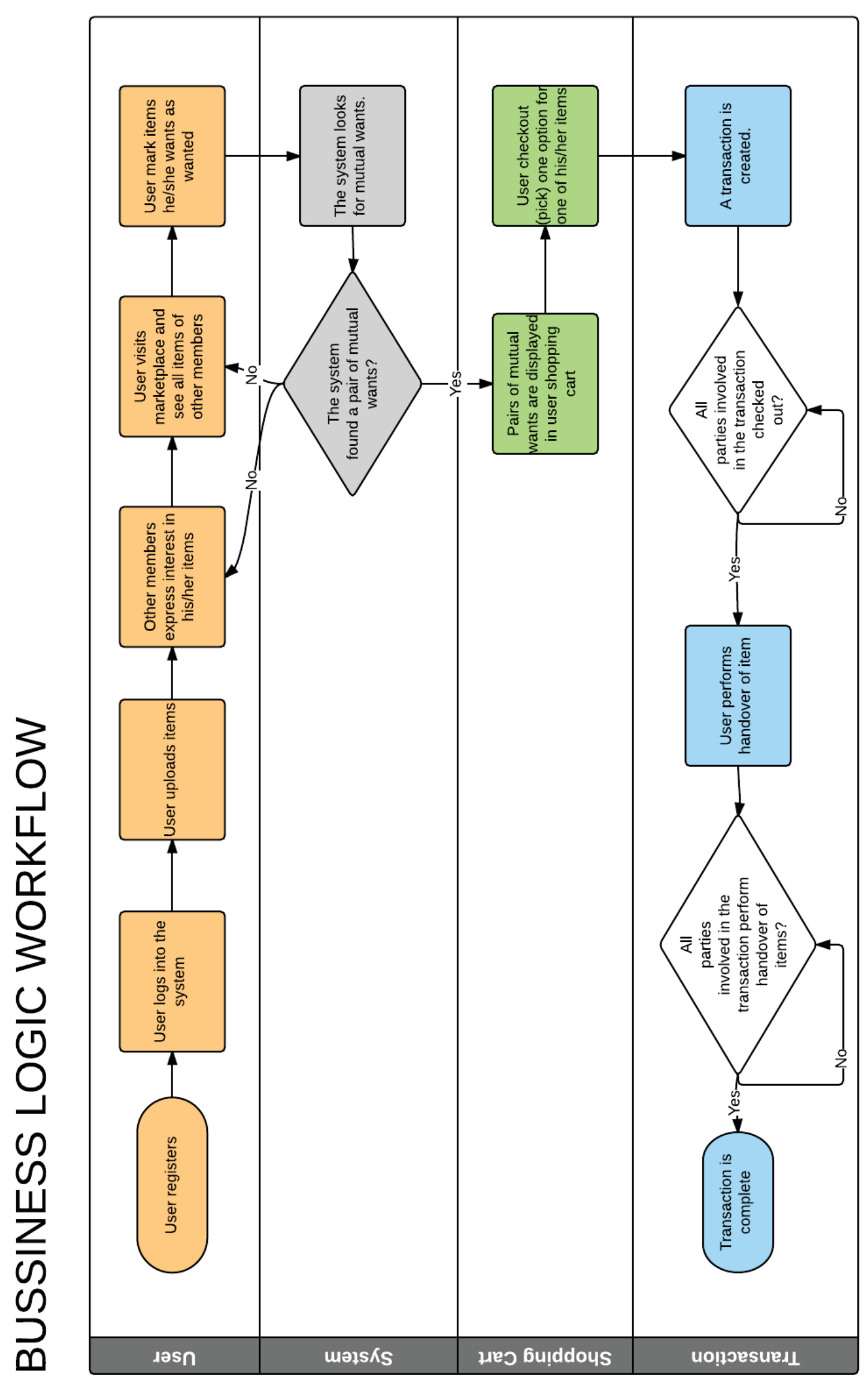

Figure 5.2: Business logic workflow 


\begin{tabular}{|l|l|l|l|l|l|}
\hline User & Gender & Age & Occupation & $\begin{array}{l}\text { Computer skills er Familiarity } \\
\text { with applications }\end{array}$ & $\begin{array}{l}\text { Experience } \\
\text { with barter }\end{array}$ \\
\hline \#1 & Male & 30 & IT Technical Lead & High & Yes \\
\hline \#2 & Female & 27 & Master Graduate & Medium & Yes \\
\hline \#3 & Male & 54 & Salesman & Entry & Yes \\
\hline $\mathbf{\# 4}$ & Male & 15 & Secondary School Student & Medium & No \\
\hline \#5 & Female & 23 & Software Tester & High & Yes \\
\hline
\end{tabular}

Table 7.1: Participant characterisation

You are given a calendar an a guitar. Your task is to exchange the calendar for a pen and the guitar for a smartphone. Register and login as a new user and perform barter. When you see your transactions marked as completed, your task has been successfully completed.

No instructions are given.

You are given a calendar an a guitar. Your task is to exchange the calendar for a pen and the guitar for a smartphone. Register and login as a new user and perform barter. When you see your transactions marked as completed, your task has been successfully completed.

Instruction: Register and login. Add two new items: calendar and guitar. Browse the marketplace, find items you want and mark them as wanted. When barter options are ready, proceed to checkout. After checkout, a transaction is created. Follow all three steps till both transaction are finished. You are given a calendar. Your task is to exchange the calendar for another item of your choice. Register and login as a new user and perform barter. When you see your transaction marked as completed, your task has been successfully completed.

Instruction: Go to Login. Register as a new user. Login with the credentials. Take a picture of your given item. Go to My Items, add the calendar as a new item. Then, go to marketplace, find any items you like and mark them as wanted. Wait for your shopping bag to fill with barter options. Then, choose one option and click checkout. You are now redirect to the transaction page. Click on the transaction a go through all 3 steps. Step 1 - you must wait for the opponent to checkout the barter option you just checked out. Step 2 proceed to the Handover Item form and confirm your handover of the item. Wait for the opponent to do the same. Step 3 - Rate the item (optional). Your transaction should be marked as completed now.

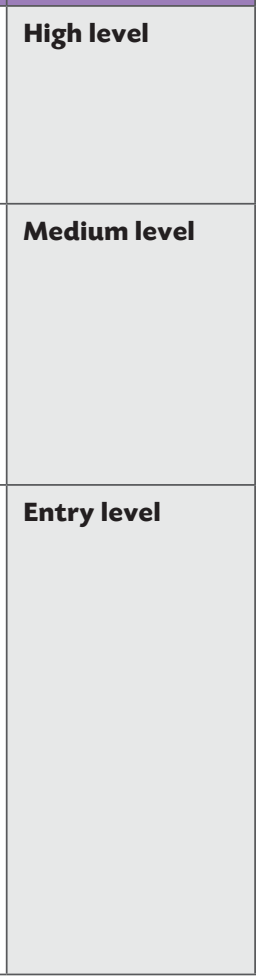

Table 7.2: Test scenarios

\begin{tabular}{|l|l|l|}
\hline $\mathbf{1 d}$ & Question & Answer Options \\
\hline $\mathbf{2}$ & $\begin{array}{l}\text { How easy or difficult was it to complete he given task? } \\
\text { completing the given tasks? }\end{array}$ & $\begin{array}{l}\text { A. Easy } \\
\text { B. Medium } \\
\text { C. Difficult }\end{array}$ \\
\hline $\mathbf{3}$ & $\begin{array}{l}\text { Compare the efficiency of barter using this e-barter system against the } \\
\text { previous method you have used to barter. }\end{array}$ & $\begin{array}{l}\text { A. Using e-barter system is more ef- } \\
\text { ficient } \\
\text { B. My previous method of barter was } \\
\text { more efficient }\end{array}$ \\
\hline $\mathbf{4}$ & $\begin{array}{l}\text { A. Unpleasant experience } \\
\text { Describe your previous experience with barter. }\end{array}$ \\
\hline $\mathbf{5}$ & $\begin{array}{l}\text { How do you compare your previous experience with barter against } \\
\text { the experience with the e-barter system? }\end{array}$ & \begin{tabular}{l} 
No predefined options \\
\hline
\end{tabular} \\
\hline
\end{tabular}


The results of the test sessions reveal the following conclusions:

- All participants were able to complete a given task. (See Figure 7.4)

- Users at entry level of computer skills (CS) and application familiarity (AF) took the most time to complete their task. The higher the level of CS and AF, the shorter time at takes a user to complete a task. (See Figure 7.5)

- $60 \%$ of participants said their task was easy to complete. (See Figure 7.6 )

- $60 \%$ of participants said the application user experience was excellent. The remainder said the user experience was good. No negative user experience occurred. (See Figure 7.7)

- All participants (80\%) said the e-barter system is more efficient for barter than their previous method of barter, one participant (20\%) did not give answer as they have never bartered before. (See Figure 7.8)

\section{SUCCESSFUL COMPLETION OF TASK}

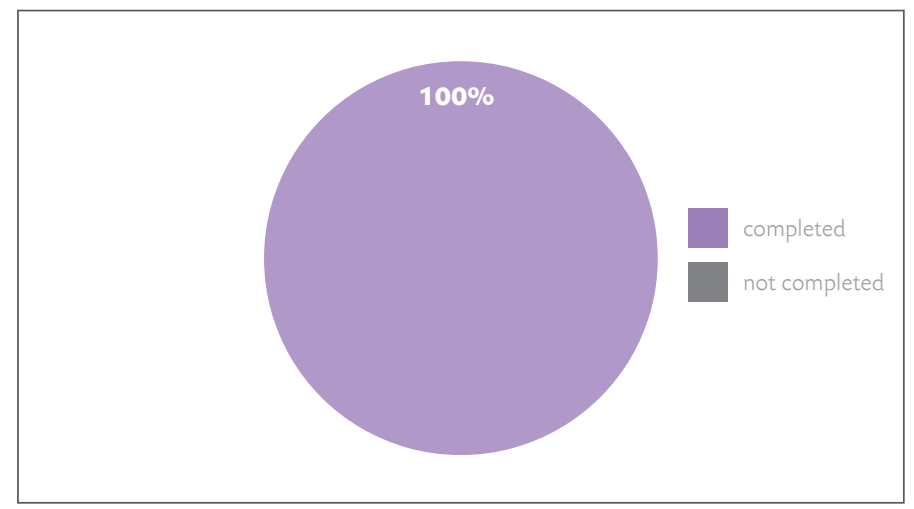

Figure 7.4: Successful completion of task

TASK COMPLETION TIME

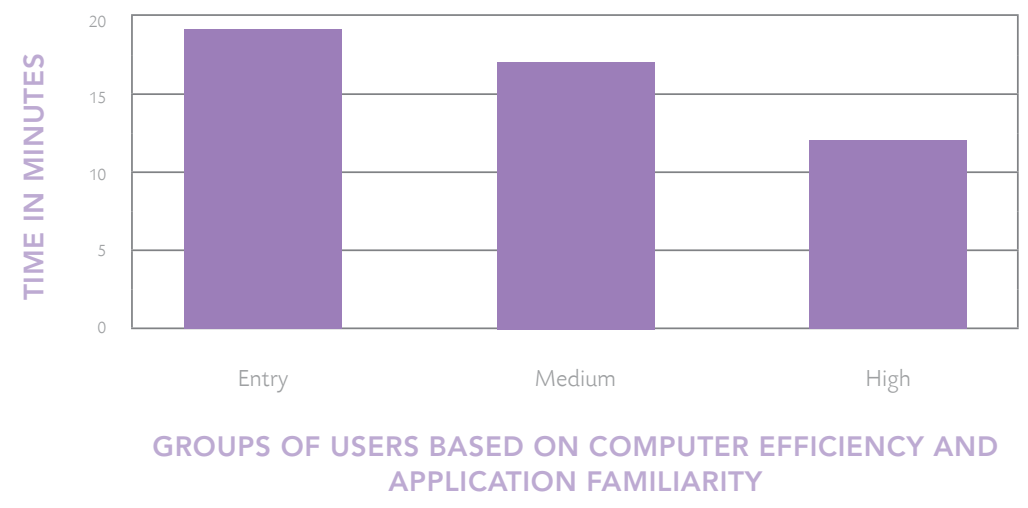

Figure 7.5: Task completion time based on different groups of users 
HOW EASY OR DIFFICULT WAS IT COMPLETE

THE GIVEN TASK?

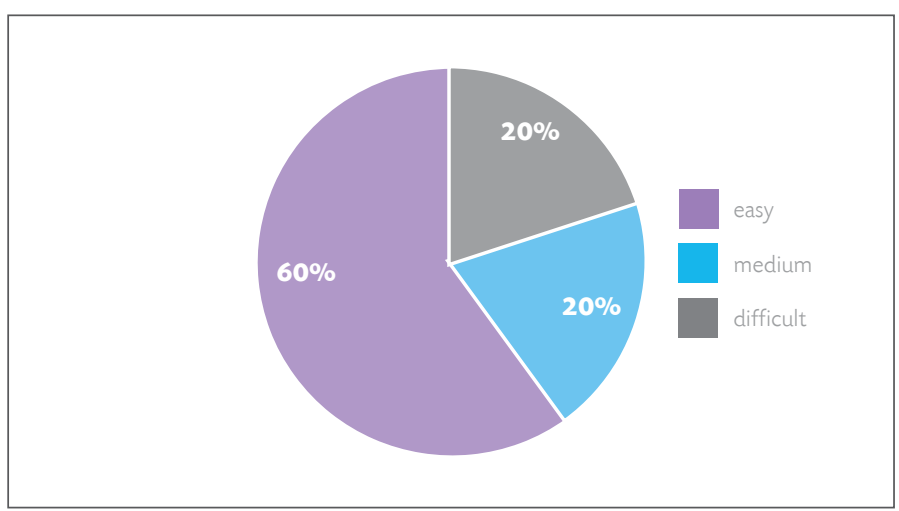

Figure 7.6: Survey question 1

HOW WOULD YOU DESCRIBE YOUR USER

EXPERIENCE USING THE APPLICATION?

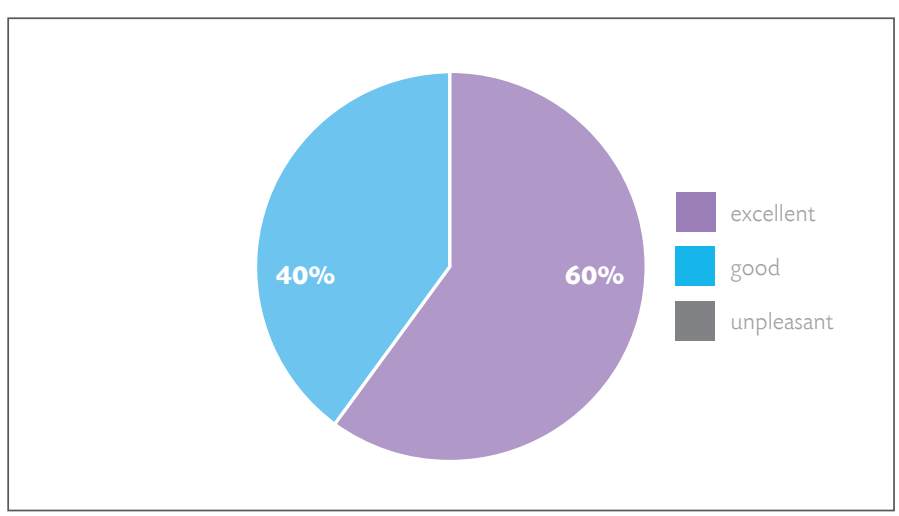

Figure 7.7: Survey question 2

COMPARE THE EFFICIENCY OF BARTER USING

THIS E-BARTER SYSTEM AGAINST THE PREVIOUS METHOD YOU HAVE USED TO BARTER.

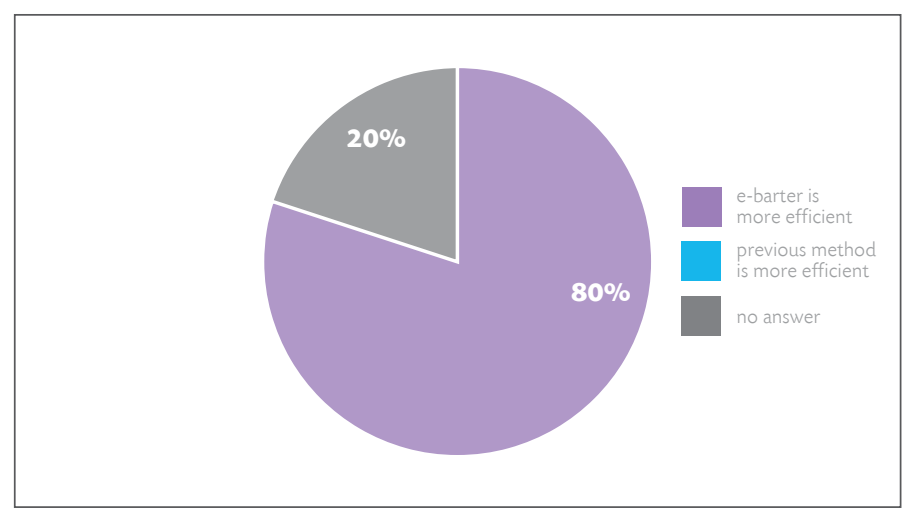

Figure 7.8: Survey question 3 


\section{CONCLUSION}

This last chapter concludes and evaluates the whole project and the artefact produced. Possible further development plans will also be discussed.

Barter, the purest form of trade, has been always challenged by its hindrances. As money came to replace barter as the dominant infrastructure of trade, barter remained unexplored. The emergence of technology, computers, and the digital age has given us new ways of solving problems. The goal of this thesis was to explore barter, the problems of barter, and use computers to bring a solution that solves some problems of barter. It is concluded that the goal was fully met. Barter has been studied, the problems of barter identified, a solution to the problem in form of a proposed model has been delivered, and an ebarter system has been implemented. The produced e-barter system solves all three identified problems of barter: problem of coincidence of wants, problem of non-feasibility of multilateral barter, and the problem of an inefficient shopping experience. Further, all the requirements for the software has been met and the e-barter system has been tested against a functional test as well as a usability test.

The produced e-barter system serves as a proof of concept, demonstrating the feasibility of the proposed theory on how to solve inefficiencies of barter. Further development would include approaching the ebarter system from a user perspective. Research shall be conducted, examining user demands, user experience, and user satisfaction in the realm of barter. By taking user input into consideration, there is proof a concept can be further developed and turned into a usable product, ready to be published to the public.

\section{REFERENCES}

Smith, A. (1776) An Inquiry Into The Nature And Causes Of The Wealth Of Nations. London: W. Strahan and T. Cadell.

Statista.com. (2015) 'B2C E-Commerce Sales Worldwide 2018', Statistic [Online]. Available at: http://www.statista. com/statistics/261245/b2c-e-commerce-sales-worldwide/ (Accessed: 16 November 2015).

Dalton, G. (1982) ' Barter', Journal of Economic Issues, [Online]. Available at: http://www.jstor.org/stable/4225147 [Accessed 23 June 2015]

Jevons, William Stanley (1875) Money and The Mechanism Of Exchange. New York: D. Appleton. 